\title{
The Role of Visual and Semantic Properties in the Emergence of Category-Specific Patterns of Neural Response in the Human Brain
}

\author{
DDavid D. Coggan, Daniel H. Baker, and (1)Timothy J. Andrews
}

DOI:http://dx.doi.org/10.1523/ENEURO.0158-16.2016

Department of Psychology and York Neuroimaging Centre, University of York, York YO10 5DD, United Kingdom

\begin{abstract}
Brain-imaging studies have found distinct spatial and temporal patterns of response to different object categories across the brain. However, the extent to which these categorical patterns of response reflect higher-level semantic or lower-level visual properties of the stimulus remains unclear. To address this question, we measured patterns of EEG response to intact and scrambled images in the human brain. Our rationale for using scrambled images is that they have many of the visual properties found in intact images, but do not convey any semantic information. Images from different object categories (bottle, face, house) were briefly presented (400 ms) in an event-related design. A multivariate pattern analysis revealed categorical patterns of response to intact images emerged $\sim 80-100$ ms after stimulus onset and were still evident when the stimulus was no longer present ( $\sim 800 \mathrm{~ms})$. Next, we measured the patterns of response to scrambled images. Categorical patterns of response to scrambled images also emerged $\sim 80-100 \mathrm{~ms}$ after stimulus onset. However, in contrast to the intact images, distinct patterns of response to scrambled images were mostly evident while the stimulus was present ( $\sim 400 \mathrm{~ms})$. Moreover, scrambled images were able to account only for all the variance in the intact images at early stages of processing. This direct manipulation of visual and semantic content provides new insights into the temporal dynamics of object perception and the extent to which different stages of processing are dependent on lower-level or higher-level properties of the image.
\end{abstract}

Key words: category; EEG; image; MVPA; object

\section{Significance Statement}

Previous studies have shown distinct spatial and temporal patterns of response to different object categories. However, the extent to which these patterns are based on lower-level visual properties compared with high-level semantic information remains unclear. To address this question, we used scrambled objects that preserve visual properties but do not convey any semantic information. We found distinct patterns of response to intact images from different object categories. Patterns of response to scrambled images from different categories emerge in a way that is similar to those of intact images but do not persist for the same duration. These findings demonstrate the relative importance of both lower-level visual and higher-level semantic properties in the neural response to objects.

\section{Introduction}

A variety of evidence has shown that spatially distinct regions of the ventral occipitotemporal cortex are selective for different categories of objects (Kanwisher, 2010).
Lesions to this region often result in difficulties in recognizing and naming specific object categories (Farah, 1990; McNeil and Warrington, 1993; Moscovitch et al., 1997). The notion that discrete areas of the temporal lobe
Received June 13, 2016; accepted June 27, 2016; First published July 21, 2016.

The authors declare no competing financial interests.
D.C., D.H.B., and T.J.A. designed research; D.C., D.H.B., and T.J.A. performed research; D.C., D.H.B., and T.J.A. analyzed data; D.C., D.H.B., and T.J.A. wrote the paper. 
are specialized for different categories of objects receives support from functional imaging studies that show that some regions in the temporal lobe are more responsive to faces than to other categories (Allison et al., 1994; Kanwisher et al., 1997). Other imaging studies have found similar category-specific responses for inanimate objects (Malach et al., 1995), scenes (Epstein and Kanwisher, 1998), and human body parts (Downing et al., 2001). Although specialized regions have only been reported for a limited number of objects, the spatial pattern of response across the entire ventral stream can distinguish a wider range of categories (Haxby et al., 2001; Kriegeskorte et al., 2008; Connolly et al., 2012; Clarke and Tyler, 2014; Rice et al., 2014).

A full understanding of object perception requires the ability to discriminate object-specific brain states with both spatial and temporal resolution. Recently, reliable patterns of neural response to images from different object categories have been shown with MEG and EEG (Carlson et al., 2011, 2013; Cauchoix et al., 2014; Cichy et al., 2014; Clarke et al., 2015). These techniques complement previous MRI studies by providing temporal information about when these categorical patterns of response emerge and how long they are sustained. Temporal properties are important, as they place constraints on models of object recognition (Mur and Kriegeskorte, 2014). Such models suggest a dynamic process in which there is a transformation from a visual representation (based on the statistics of the image) to a semantic representation (reflecting the meaning of the object; Clarke and Tyler, 2014). It is thought that the initial component of the response reflects fast feedforward processing that is related to visual properties, whereas later patterns reflect recurrent processing that might be related to semantic properties of the stimulus (Lamme and Roelfsema, 2000; Hochstein and Ahissar, 2002; Bar et al., 2006; DiCarlo and Cox, 2007).

The aim of this study was to investigate the relative importance of visual and semantic properties of objects in the emergence of categorical patterns of neural response. However, a fundamental problem in this endeavor is that the visual and semantic properties of objects often covary, making it difficult to resolve the relative contribution of these sources of information to patterns of neural response. So, it is not clear from many previous studies whether the distinct patterns of response to different object categories reflect visual or semantic properties (Carlson et al., 2011, 2013; Cauchoix et al., 2014; Cichy

This research was supported in part by the Wellcome Trust (Grant $100004440105624)$ through the Centre for Chronic Diseases and Disorders (C2D2) at the University of York.

Correspondence should be addressed to either Timothy J. Andrews or Daniel H. Baker, Department of Psychology and York Neuroimaging Centre, University of York, York YO10 5DD, UK. E-mail: timothy.andrews@york.ac.uk or daniel.baker@york.ac.uk.

DOI:http://dx.doi.org/10.1523/ENEURO.0158-16.2016

Copyright (C) 2016 Coggan et al.

This is an open-access article distributed under the terms of the Creative Commons Attribution 4.0 International, which permits unrestricted use, distribution and reproduction in any medium provided that the original work is properly attributed. et al., 2014). In a recent MEG study, Clarke et al. (2015) addressed this issue by showing that the categorization of objects based on the neural response could be predicted by the visual properties of the image. However, they also found that accuracy could enhanced by including semantic properties, particularly at later stages of processing. Although this suggests that visual and semantic properties are both important for the neural representation of objects, this approach is not able to show a causal link.

To address this issue, we measured patterns of EEG response to intact images from different object categories, as well as versions of these images that had been phase scrambled on a global or local basis. Our rationale for using scrambled images is that they have many of the visual properties found in intact images, but they do not convey any semantic information (Coggan et al., 2016). This allows us to determine the extent to which the preserved visual properties contribute to the neural representation of objects in the absence of any semantic content. The comparison between the locally scrambled and globally scrambled images also allows us to explore the importance of spatial image properties, which are preserved in the locally scrambled condition. In a recent fMRI study, we found similar spatial patterns of response to intact and scrambled images across the ventral visual pathway (Coggan et al., 2016). This study demonstrated the importance of low-level visual properties in the patterns of response in the ventral visual pathway. By comparing the similarity of the responses to intact and scrambled images using EEG, we aim to determine the relative contribution of visual properties to categorical patterns of response at different time points.

\section{Materials and Methods}

\section{Stimuli}

On hundred five images of three object categories (face, bottle, and house) were taken from an object-image stimulus set (Rice et al., 2014). All images were grayscale, were superimposed on a middle gray background, and had a resolution of $400 \times 400$ pixels (Fig. 1). For each of these original images, two different phase-scrambled versions were generated. A global-scrambling method involved a typical Fourier scramble (i.e., keeping the global power of each two-dimensional frequency component constant while randomizing the phase of the components). A local-scrambling method involved windowing the original image into an $8 \times 8$ grid and applying the phase scramble to each $50 \times 50$ pixel window independently. In a previous study (Coggan et al., 2016), we showed that these scrambling methods effectively remove any semantic or categorical content in the images. Stimuli were presented using a gamma-corrected VIEWPixx display (VPixx Technologies) with a resolution of $1920 \times 1200$ pixels and a refresh rate of $120 \mathrm{~Hz}$. Images were viewed at a distance of $\sim 57 \mathrm{~cm}$ and subtended a retinal angle of $8^{\circ}$.

\section{Participants}

Twenty participants ( 3 males; mean age, 20.6 years; SD, 2.6 years) with normal or corrected-to-normal vision took 

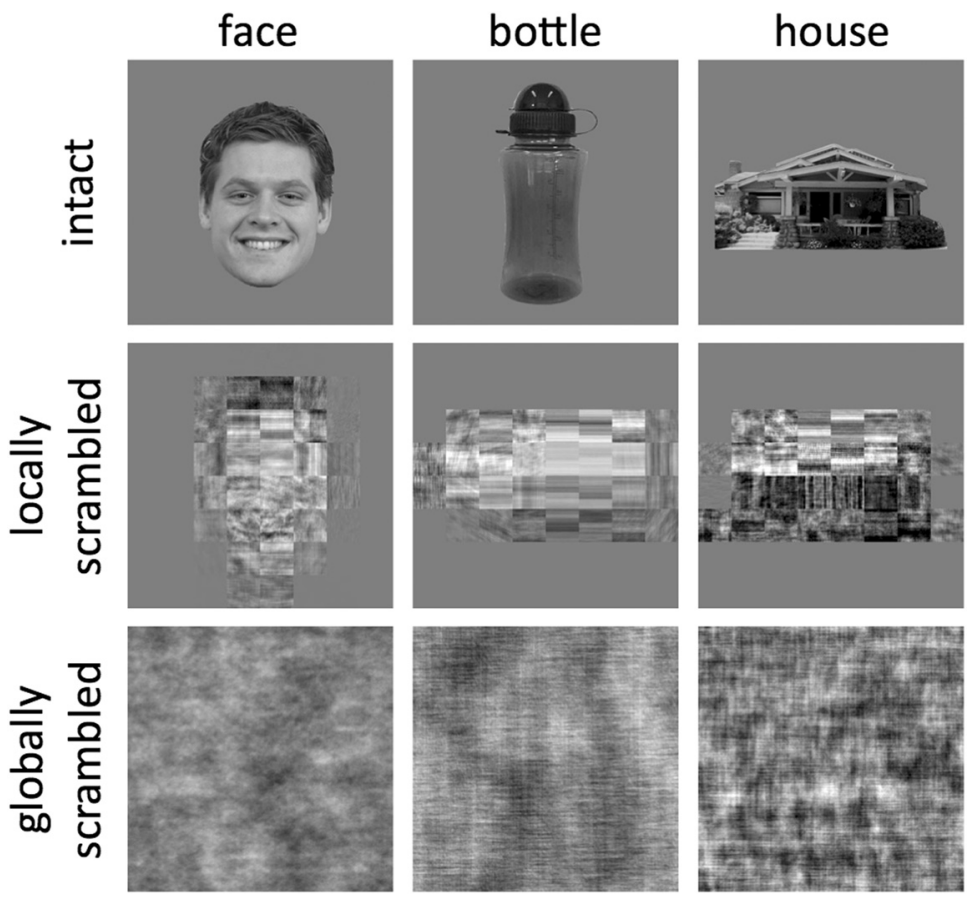

Figure 1. Exemplars of intact, locally scrambled, and globally scrambled images from the different object categories.

part in the experiment. Participants gave written, informed consent. The study was approved by the University of York Department of Psychology Ethics Committee. The data for one participant (female) were removed from the analysis due to partial data loss.

\section{Design and procedure}

The experiment involved the following three runs: the first run contained globally scrambled images; the second run contained locally scrambled images; and the third run contained intact images. Therefore, participants were unaware of the object categories in our stimulus set prior to viewing the scrambled images. Each run contained 35 blocks. There were 10 trials in each block. In each trial, an image from one of the three object categories was presented for $400 \mathrm{~ms}$. There was a jittered intertrial interval that had a mean duration of $1 \mathrm{~s}$ and an SD of $200 \mathrm{~ms}$. The duration of the interblock interval was $3 \mathrm{~s}$. Participants fixated a cross in the center of the screen between trials. To maintain attention, participants were instructed to click a mouse whenever a red dot appeared on an image. One image in each block contained a red dot. Self-timed rests were taken between runs.

\section{EEG recording}

EEG waveforms were recorded from 64 scalp locations laid out according to the 10/20 system in a WaveGuard cap (ANT Neuro). Data from each electrode were referenced against a whole-head average. We also monitored blinks through bipolar electro-oculogram (EOG) electrodes placed above and below the left eye. Signals were amplified and digitized at $1000 \mathrm{~Hz}$ and recorded using the ANT Neuroscan software (ANT Neuro). Stimuluscontingent triggers were sent from the VIEWPixx device to the EEG amplifier using a 25-pin parallel port with microsecond-accurate synchronization to the display refresh sequence. The PsychToolbox routines (Brainard, 1997; Pelli, 1997) running in Matlab were used to control the display hardware and to send triggers.

\section{EEG preprocessing}

The EEG traces from each run were concatenated and bandpass filtered between 0.01 and $30 \mathrm{~Hz}$ prior to epoching. Blink artifacts were corrected using independent components analysis (ICA). This involved running ICA across data from all electrodes, including the vertical EOG, and manually selecting the components that captured blink artifacts. These components were then subtracted from the EEG trace at each electrode site according to their weighting. This approach meant that no trials were rejected. The EEG trace was then divided into epochs ranging from $200 \mathrm{~ms}$ before stimulus onset to 800 ms after stimulus onset. All trials containing a red dot were removed prior to further analysis.

\section{EEG MVPA analysis}

All data processing was performed in Matlab using custom scripts. To measure the spatial patterns of EEG response for each participant, trials were collapsed into mean ERPs for odd and even trials for each condition and at each electrode site. These condition-averaged ERPs were then baselined by subtracting the mean amplitude during the $200 \mathrm{~ms}$ prior to stimulus onset (across both odd and even trials) from the response at each time point. From these ERPs, a 64-value vector representing the spatial pattern of response across all electrodes was extracted for odd and even trials for each object category at each time point. 
Pattern vectors were normalized within each participant using the following method. First, vectors were selected from one time point and one image type. This gave a total of six patterns (odd/even $\times$ face/bottle/house). For each electrode site, the mean amplitude across all six patterns was subtracted from its amplitude in each pattern. This process was repeated for each image type at each time point.

To see whether different object categories evoke distinct patterns of EEG response, we ran a correlationbased MVPA separately for each image type and time point (Fig. 2A). This involved measuring the correlation between pattern vectors within and among the three object categories. For within-category correlations (e.g., face vs face), we measured the correlation between odd and even trials. For between-category correlations (e.g., bottle vs house), we used the mean correlation between odd trials of the first category and even trials of the second, and between even trials of the first category and odd trials of the second. The distinctiveness of the patterns of EEG response was then measured by subtracting between-category correlations from within-category correlations. The $95 \%$ confidence intervals (Cls) for this difference were then obtained by bootstrapping across participants. Points at which different object categories evoked significantly distinct patterns of EEG response were defined by the lower confidence interval being $>0$.

To measure the similarity between responses to intact and scrambled images from the same object category, we first collapsed patterns across odd and even trials to create one pattern per condition per time point. We then correlated the patterns of response at each time point separately for the intact locally scrambled and the intact globally scrambled contrasts for each category. A group mean was calculated across categories, and 95\% confidence intervals were obtained by bootstrapping across participants.

To determine whether the response to intact images could be explained by the response to scrambled images, we calculated a noise ceiling. This estimates that maximum correlation that could be expected. The noise ceiling was calculated by measuring the correlation between the responses at odd and even trials within each category in the intact condition. At the individual level, we take a mean of the within-category correlations (face-face, bottle-bottle, house-house) for each time point. We then average across subjects to obtain one noise ceiling estimate at each time point. Time points at which this value fell within the $95 \% \mathrm{Cl}$ for the correlation between intact and scrambled images demonstrate when all the variance in the intact images was explained by the scrambled images.

The correlation-based method was complemented with a classification-based approach involving a support vector machine, producing similar results. To see whether different object categories evoked distinct patterns of response, classification was performed separately for each participant, image type, and time point (see Fig. 6). First, patterns of EEG response were extracted for each trial of each category. Two "training" patterns and one "testing" pattern for each category were generated by randomly dividing the 105 trials into three equal sets and taking an average. A support vector machine was then trained on the six training patterns, and tested on the three testing patterns. This procedure was repeated 100 times, with different subsets of trials used for training and testing in each iteration. To see whether similar patterns of response were evoked by intact and scrambled images from the same category, the classifier was altered so that test patterns were substituted with those from another image type. This was performed for each pairwise contrast between image types, and accuracy was averaged across both directions (e.g., train on intact, test on locally scrambled; and train on locally scrambled, test on intact).

Finally, to examine transient and persistent neural activity in response to each condition, we conducted a temporal cross-correlation. This involved measuring the correlation between response patterns for odd and even trials for the same condition, iterating over each possible pair of time points. Correlations were represented in a $1000 \times 1000$ similarity matrix, and data were averaged across the positive diagonal. Matrices were then collapsed across categories to give one matrix per image type.

\section{Results}

First, we asked whether different intact object categories produced distinct spatial patterns of EEG response (Fig. 2). To address this question, we compared the similarity of patterns of response to images from the same category (e.g., face vs face) with the similarity of patterns to images of different categories (e.g., face vs house). Categorical patterns of response were demonstrated when the withincategory correlations were significantly greater than the between-category correlations. Categorical patterns of response to intact images emerged $80 \mathrm{~ms}$ after stimulus onset. The patterns were maximally distinct at $\sim 150 \mathrm{~ms}$ and persisted until at least $800 \mathrm{~ms}$ (Fig. 2B). A classificationbased approach was then used to complement the correlation-based method. In this analysis, a classifier was trained on a subset of the data and tested on the remaining data. This showed a pattern that was similar to that derived from the correlation-based analysis. Accuracy above the level of chance emerged $80 \mathrm{~ms}$ after stimulus onset, peaked at about $150 \mathrm{~ms}$, and persisted until $800 \mathrm{~ms}$ (Fig. 3A).

To measure the extent to which these category-specific patterns of response were based on lower-level visual properties, we first asked whether locally scrambled and globally scrambled images also produced distinct category-specific patterns of EEG response using both the correlation-based (Fig. 2C,D) and classification-based (Fig. 3B,C) analyses. Distinct category-specific patterns of response for locally scrambled images emerged at $\sim 80$ ms after stimulus onset. They were maximally distinct at $\sim 110 \mathrm{~ms}$ and persisted until $\sim 400-500 \mathrm{~ms}$. Distinct category-specific patterns of response for globally scrambled images emerged at $\sim 100 \mathrm{~ms}$ after stimulus onset. They were maximally distinct at $\sim 190 \mathrm{~ms}$ and persisted until $\sim 300 \mathrm{~ms}$. 
A

64-channel EEG ERP data

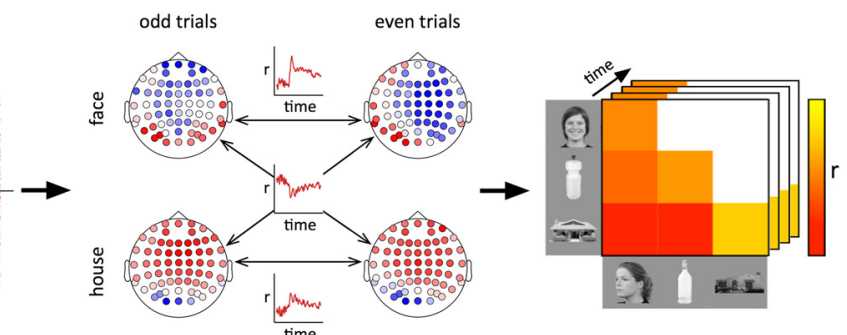

B

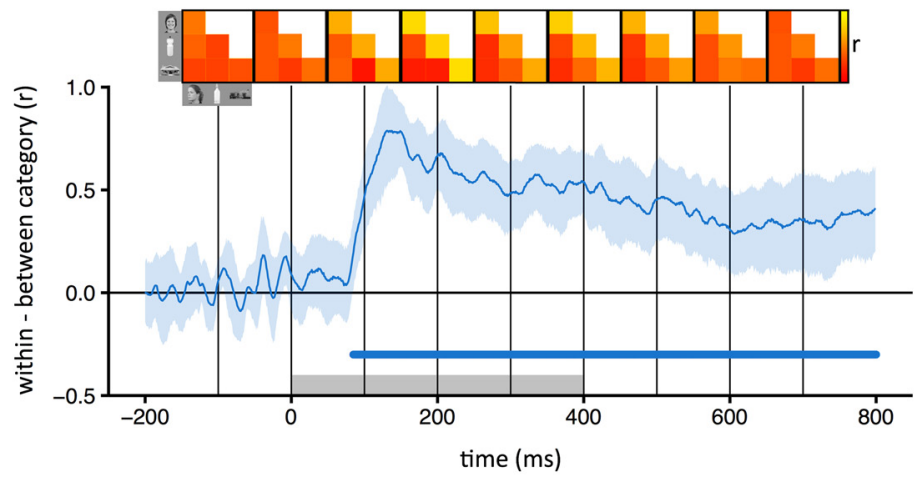

C

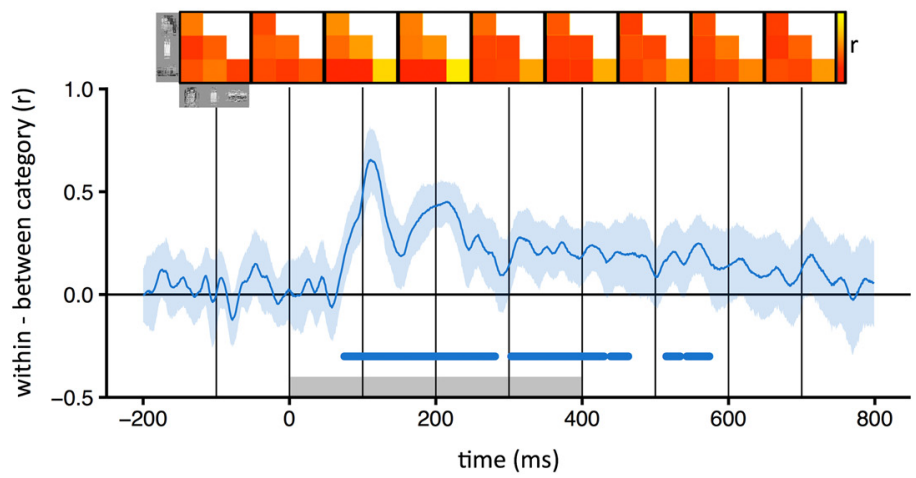

D

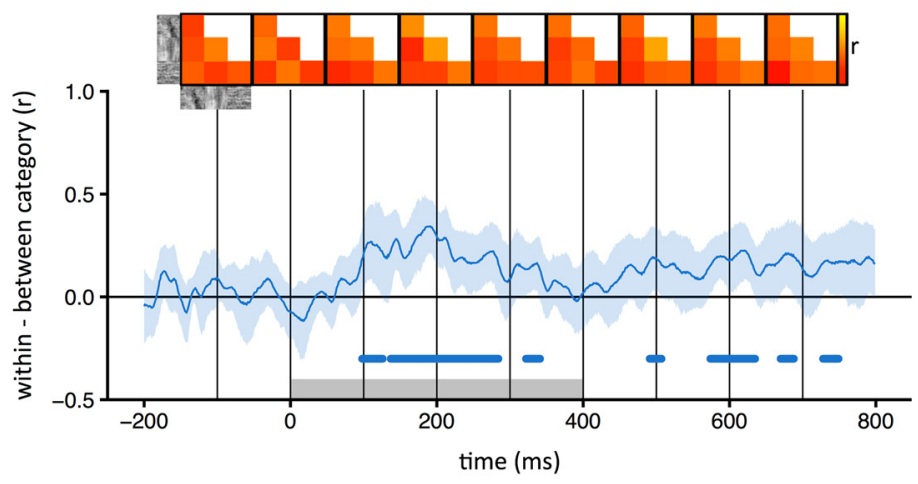

Figure 2. Category-specific patterns of EEG response to intact and scrambled images. $\boldsymbol{A}$, For each time point, normalized patterns of response to odd and even trials of each category were compared across 64 electrodes. The correlation coefficients were then represented in a similarity matrix for that time point. Distinct category-specific patterns of response were defined by higher within-category (e.g., face-face) compared to between-category (e.g., face-bottle) correlations. $\boldsymbol{B}-\boldsymbol{D}$, Correlation time-courses are shown for the intact $(\boldsymbol{B})$, locally scrambled $(\boldsymbol{C})$, and globally scrambled $(\boldsymbol{D})$ image types. The shaded region represents $95 \%$ Cls obtained by bootstrapping across participants. Group mean correlation matrices at $100 \mathrm{~ms}$ intervals are shown above the plot. Gray 
continued

box at the base of the plot represents the time points at which the stimulus was present. Blue bar at the base of the plot represents time points at which the lower bound of the $\mathrm{Cl}$ is $>0$, indicating significantly higher within-category correlations than betweencategory correlations.

Although distinct patterns of response were evident to scrambled images from different categories (i.e., withincategory $>$ between-category correlations), it is not clear whether the patterns were similar to those elicited from the intact images. To address this question, we correlated patterns of response to the same object category across
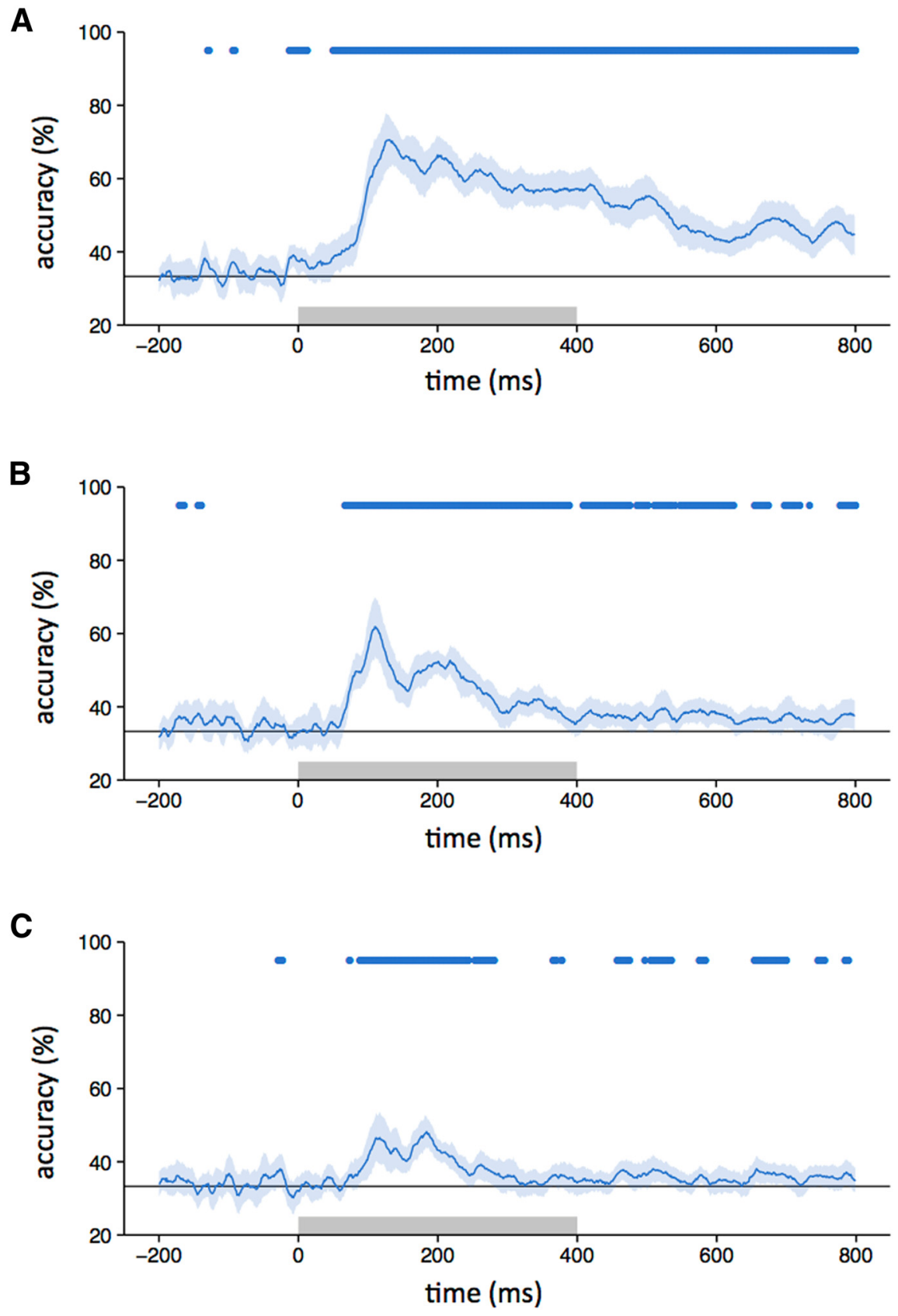

Figure 3. $\boldsymbol{A}-\boldsymbol{C}$, Classifier accuracy for between-category discrimination (blue line) with intact $(\boldsymbol{A})$, locally scrambled (B), and globally scrambled $(\boldsymbol{C})$ images (chance $=33 \%$, gray line). The blue-shaded regions represent $95 \%$ Cls obtained through bootstrapping across participants. The blue bar at the top of the plot represents time points at which the lower bound of the $\mathrm{Cl}$ is above chance. The gray box on the axes of the plot represents the stimulus duration. 


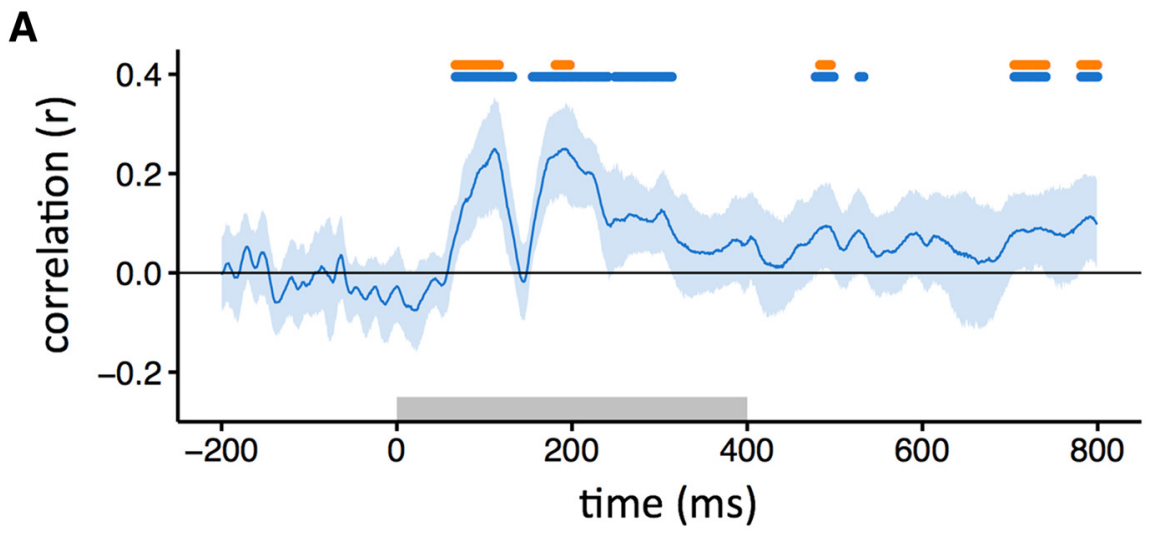

B

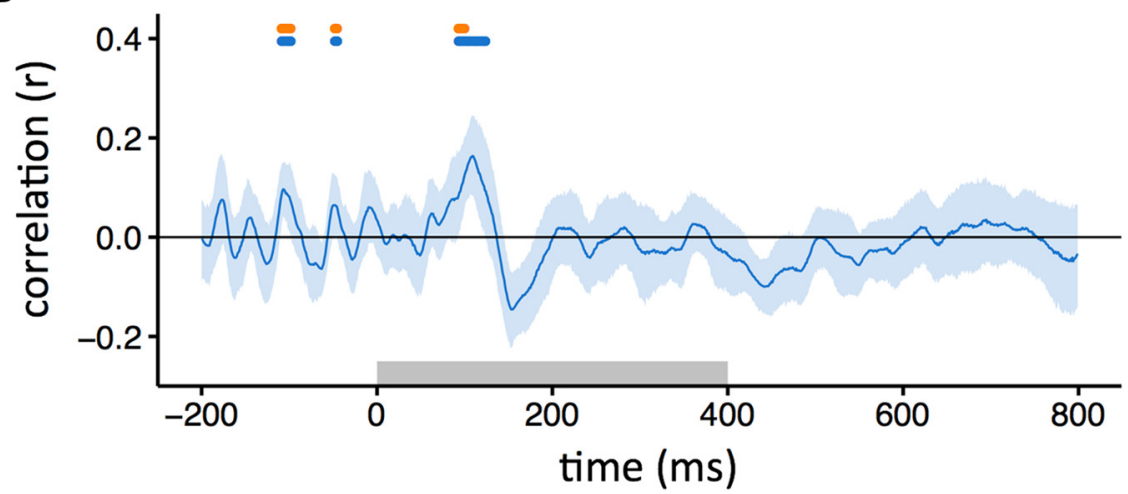

Figure 4. Similarity between patterns of EEG response to intact images and locally scrambled $(\boldsymbol{A})$ or globally scrambled $(\boldsymbol{B})$ images from the same object category. Blue-shaded regions represent $95 \%$ Cls across participants. The blue bar at the top of the plot indicates time points at which the correlation is significantly $>0$. The orange bar indicates the time points at which the correlation is not significantly different from the noise ceiling. The gray box represents the stimulus duration.

different levels of scrambling at different time points. Figure $4 A$ (blue horizontal bar) shows that the correlation between intact and locally scrambled images became significant at $\sim 80 \mathrm{~ms}$ after stimulus onset, and peaked at $\sim 110$ and $190 \mathrm{~ms}$. The percentage duration that the locally scrambled patterns were correlated with the intact patterns was greater during the stimulus period $(0-400$ $\mathrm{ms}, 27 \%$ ) compared with the poststimulus period (400$800 \mathrm{~ms}, 10 \%)$. A similar pattern of results was evident when we trained a classifier on intact or locally scrambled images and then tested on locally scrambled or intact images, respectively (Fig. 5A). The duration of accuracy above the level of chance with the locally scrambled and intact conditions was similar during the stimulus period $(0-400 \mathrm{~ms}, 40 \%)$ and the poststimulus period (400-800 ms, $49 \%)$.

Next, we explored the similarity between the intact and globally scrambled images (Figs. $4 B, 5 B$ ). The correlation between responses to intact and globally scrambled images became significant (blue horizontal bar) $\sim 90 \mathrm{~ms}$ after stimulus onset, peaked at $\sim 110 \mathrm{~ms}$, and persisted until $\sim 120 \mathrm{~ms}$. The percentage duration that the locally scrambled patterns were correlated with the intact patterns was greater during the stimulus period $(0-400 \mathrm{~ms}$, $4 \%)$ compared with the poststimulus period (400-800 $\mathrm{ms}, 0 \%$ ). A similar pattern of results was evident when we trained a classifier on intact or locally scrambled images and then tested it on locally scrambled or intact images, respectively (Fig. 5A). The duration of accuracy above the level of chance with the locally scrambled and intact conditions was greater during the stimulus period $(0-400$ $\mathrm{ms}, 4 \%)$ compared with the poststimulus period (400$800 \mathrm{~ms}, 0 \%)$.

To directly compare the similarity between intact images and either locally scrambled or globally scrambled images, the average correlation (Fig. 4) or accuracy (Fig. 5) was compared across individuals. The average correlation between intact and locally scrambled images was significantly higher than the correlation between intact and globally scrambled images $\left(t_{(18)}=3.29, p<0.005\right)$. Similarly, the average accuracy (Fig. 5) with intact and locally scrambled images was significantly higher than with intact and globally scrambled images $\left(t_{(18)}=5.34\right.$, $p<0$.0001).

We then asked whether the explainable variance in intact responses was fully accounted for by the responses to scrambled images, given the level of noise in the data. This was achieved by calculating a noise ceiling (Nili et al., 2014). This involved measuring the correlation to intact images from the same category across odd and even trials of the same category. The noise ceiling was not fixed, but varied across time. We then determined 

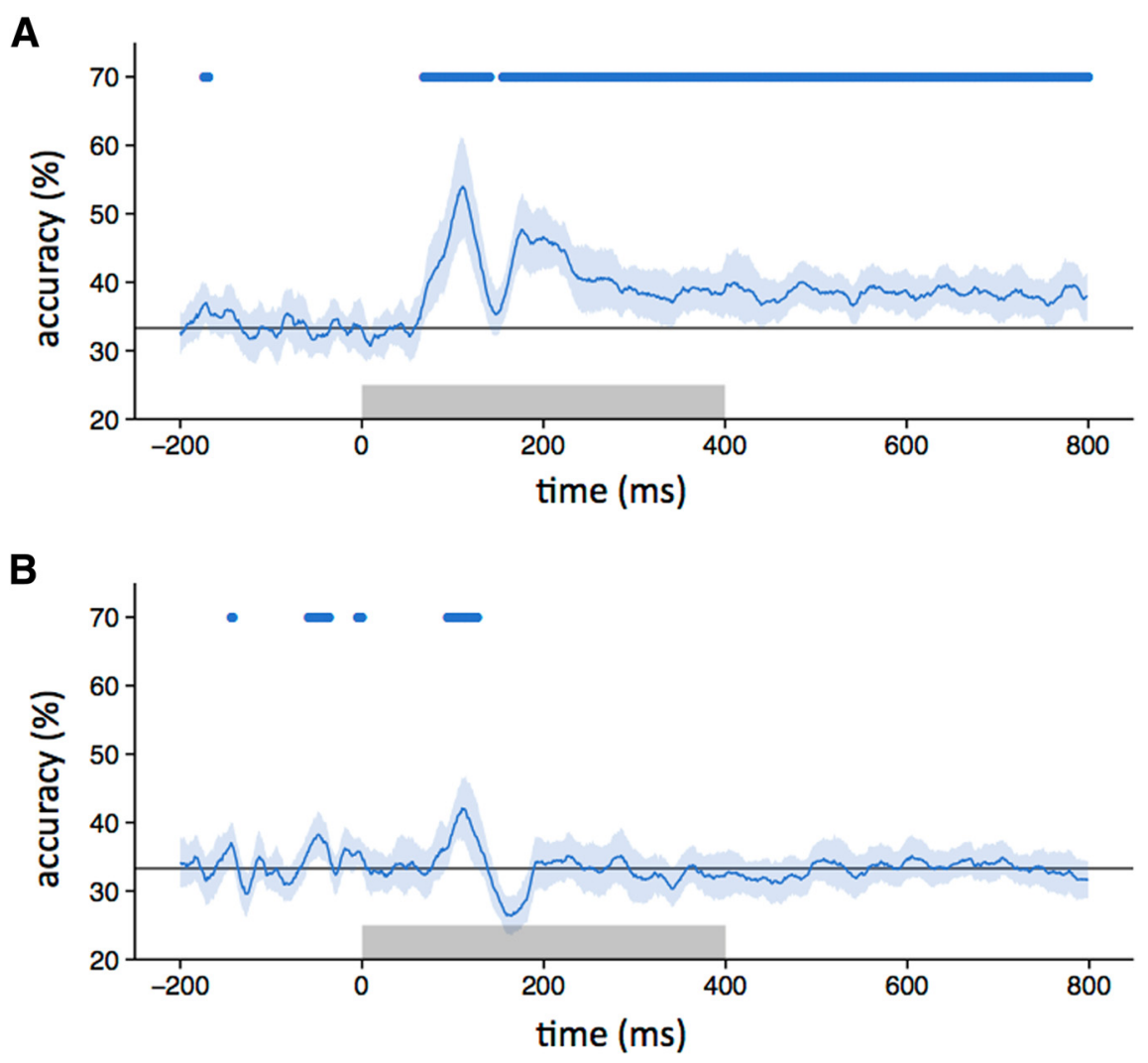

Figure 5. Classifier performance across different image types. $\boldsymbol{A}$, Accuracy in classifying responses to either intact or locally scrambled images when trained on locally scrambled or intact images, respectively. $\boldsymbol{B}$, Accuracy in classifying responses to either intact or globally scrambled images when trained on globally scrambled or intact images, respectively. The blue line indicates classifier accuracy across time, with shaded regions representing 95\% Cls obtained through bootstrapping across participants. The blue bar at the top of the plot represents the time points at which the lower bound of the $\mathrm{Cl}$ is above chance. The gray box shows stimulus duration.

whether the correlation between intact and scrambled images was not significantly different from the noise ceiling for each time point. For locally scrambled images, the $95 \%$ Cls of the correlations overlapped until $\sim 120 \mathrm{~ms}$ after stimulus onset (Fig. 4A). The percentage duration that the locally scrambled patterns were not significantly different from the noise ceiling was similar during the stimulus period $(0-400 \mathrm{~ms}, 9 \%)$ and the poststimulus period (400-800 ms, 9\%). For globally scrambled images, the confidence intervals overlapped until $\sim 100 \mathrm{~ms}$ after stimulus onset (Fig. 4B). The percentage duration that the globally scrambled patterns were not significantly different from the noise ceiling was greater during the stimulus period $(0-400 \mathrm{~ms}, 1 \%)$ compared with the poststimulus period $(400-800 \mathrm{~ms}, 0 \%)$.

Finally, we investigated the stability of the categoryspecific patterns of response for each image manipulation (Cichy et al., 2014). This involved measuring the correlation between the patterns of EEG response within each condition across different time points. The results were then averaged across categories for each image type and represented in time-time similarity matrices (Fig. 6). Here, the diagonal for intact images corresponds to the noiseceiling estimate used in Figure 4. For intact images, the pattern of response from 100 to 150 ms was positively correlated with patterns found from $\sim 250$ to $600 \mathrm{~ms}$. The continuation of this neural activity far beyond stimulus offset suggests that this does not reflect prolonged visual input during image presentation. The locally scrambled matrix shows no evidence of persistent neural activity as seen in the intact matrix, but does exhibit transient neural activity between $\sim 100$ and $250 \mathrm{~ms}$ after stimulus onset. Interestingly, time point combinations of $\sim 150$ and $\sim 200$ ms show negative correlations, suggesting a polarity reversal in the potentials between these latencies. The globally scrambled matrix shows weak correlations across all combinations of time points.

\section{Discussion}

The aim of this study was to determine the contribution of lower-level visual and higher-level semantic properties to the emergence of categorical patterns of neural response. To address this question, we compared patterns of EEG response to intact and scrambled images from different object categories. Scrambled images were used because they contain visual properties similar to those of intact images but do not convey any semantic information (Coggan et al., 2016). Our results show similar categoryspecific patterns of response at early stages of processing. However, these patterns were sustained for a longer time 

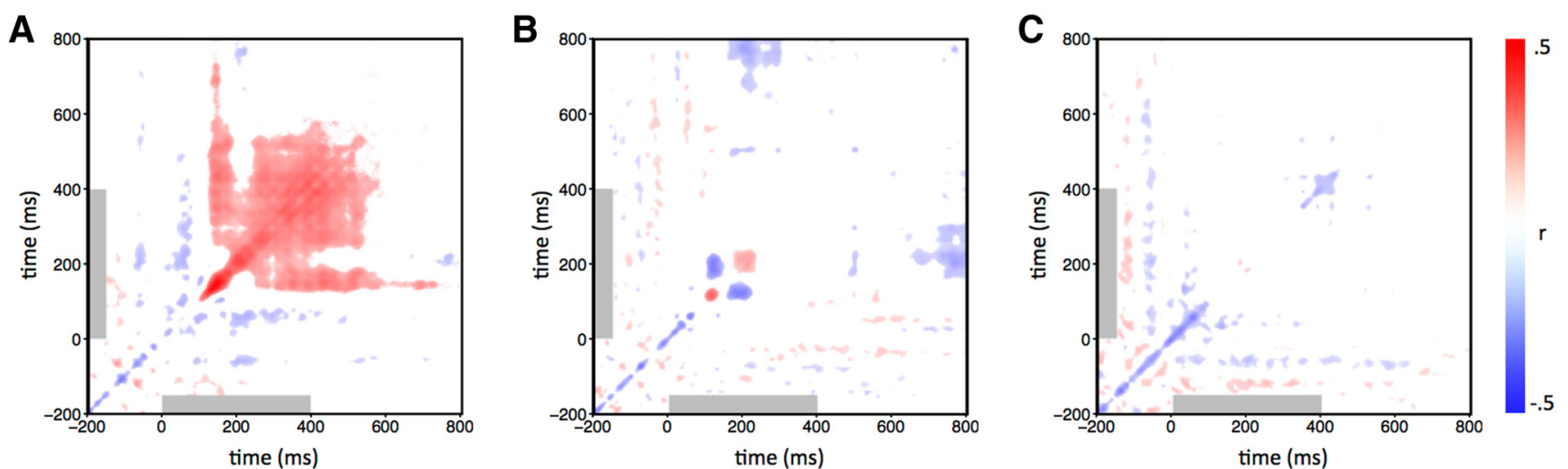

Figure 6. Temporal cross-correlation matrices for each image type. Responses to trials of the same condition were correlated over each combination of time points. $\boldsymbol{A}-\boldsymbol{C}$, Correlations were collapsed across categories to give one matrix per image type [intact $(\boldsymbol{A})$, locally scrambled $(\boldsymbol{B})$, globally scrambled $(\boldsymbol{C})]$. The colorbar represents Pearson's correlation coefficients. Matrices were thresholded by obtaining $95 \%$ Cls at each coordinate by bootstrapping across participants. Coordinates at which these intervals overlapped with 0 are shown in white. The gray box represents the stimulus duration.

with intact images compared with scrambled images. These results show the importance of visual properties in the emergence of categorical patterns of response, but also show the importance of semantic properties in the recurrent processing that sustains these patterns.

The emergence of category-specific patterns of EEG response to intact images is comparable to previous studies using MEG that found that categorical distinctions can be decoded prior to $100 \mathrm{~ms}$ after stimulus onset and become maximally distinct at $\sim 140 \mathrm{~ms}$ (Carlson et al., 2013; Cauchoix et al., 2014; Cichy et al., 2014). However, most previous studies have not directly determined whether these patterns of response reflect lower-level visual properties or higher-level semantic properties of the image. Recently, Clarke et al. (2015) addressed this issue with MEG showing that visual properties can explain patterns of response to different categories of objects. However, they also showed that the semantic properties of objects were able to explain additional variance in the pattern of response, particularly at later stages of the response. In our study, we were also able to show that the patterns of response to images from different object categories are driven predominantly by the lower-level visual properties at early stages of visual processing (up to $150 \mathrm{~ms}$ ). Visual properties were also able to partially account for the variance in the response to intact images at later stages of processing.

Patterns of response to intact images were correlated more strongly and for a longer period of time with responses to locally scrambled images than with globally scrambled images. One key difference between these two conditions is that the spatial properties, such as the shape (or spatial envelope) of the image, are somewhat preserved in the locally scrambled images, but not in the globally scrambled images. In a recent fMRI study, we showed that the spatial pattern of response in the ventral stream to different categories of intact objects was more similar to the pattern elicited by locally scrambled objects compared with globally scrambled objects. The greater similarity between responses to intact locally scrambled images is consistent with previous studies that have shown a modulatory effect of spatial properties on patterns of response in the ventral visual pathway (Levy et al., 2001; Golomb and Kanwisher, 2012; Silson et al., 2015; Bracci and Op de Beeck, 2016; Watson et al., 2016).

Although lower-level image properties account for the majority of the variance in responses to intact images at early stages, there remains a significant amount of variance to be explained at later stages of processing. For example, although category-specific patterns of response to intact images persisted well beyond the duration of the stimulus, patterns of response to scrambled images were evident only when the stimulus was present. The persistence of these neural responses to intact images suggests an important role for recurrent processing of the image, which is likely to be driven by top-down semantic representations (Lamme and Roelfsema, 2000; DiCarlo and Cox, 2007; Kriegeskorte et al., 2008; Naselaris et al., 2009; Connolly et al., 2012; Mur and Kriegeskorte, 2014). Indeed, Clarke et al. (2015) showed that accuracy in categorization using MEG data was enhanced by combining visual and semantic models.

It is also possible that differences in the patterns of response between intact and scrambled images reflect sensitivity to image properties that are disrupted by either scrambling process. An important property of natural images is that they contain strong statistical dependencies, such as location-specific combinations of orientation and spatial frequency corresponding to image features such as edges (Marr and Hildreth, 1980). Indeed, the character and extent of these statistical dependencies is likely to be diagnostic for different classes of images (O'Toole et al., 2005; Rice et al., 2014). The scrambling procedure disrupts many of the statistical relationships between the elements. So, it is possible that image manipulations that can preserve these higher-level visual properties (Freeman and Simoncelli, 2011) might generate responses that are more similar to the intact images. Indeed, it is possible that neural representations underlying higher-level visual 
properties and the corresponding semantic properties that they convey may be the same.

In conclusion, we have found that distinct categoryspecific patterns of neural response emerge at $\sim 80 \mathrm{~ms}$ after stimulus onset and can persist for at least $800 \mathrm{~ms}$. Using scrambled images, we show that early stages of these category-specific patterns can be explained by lower-level image properties. However, the differences in the neural responses to intact and scrambled images at later stages of processing also reveal the importance of higher-level semantic properties.

\section{References}

Allison T, Ginter H, McCarthy G, Nobre AC, Puce A, Luby M, Spencer DD (1994) Face recognition in human extrastriate cortex. J Neurophysiol 71:821-825. Medline

Bar M, Kassam KS, Ghuman AS, Boshyan J, Schmidt AM, Dale AM, Hämäläinen MS, Marinkovic K, Schacter DL, Rosen BR, Halgren E (2006) Top-down facilitation of visual recognition. Proc Natl Acad Sci U S A 103:449-454. CrossRef Medline

Bracci S, Op de Beeck H (2016) Dissociations and associations between shape and category representations in the two visual pathways. J Neurosci 36:432-444. CrossRef Medline

Brainard DH (1997) The Psychophysics toolbox. Spat Vis 10:433436. Medline

Carlson TA, Hogendoorn H, Kanai R, Mesik J, Turret J (2011) High temporal resolution decoding of object position and category. J Vis 11(10):9 1-17. CrossRef Medline

Carlson TA, Tovar D, Alink A, Kriegeskorte N (2013) Representational dynamics of object vision: the first $1000 \mathrm{~ms}$. J Vis 13(10):1 1-19. CrossRef Medline

Cauchoix M, Barragan-Jason G, Serre T, Barbeau EJ (2014) The neural dynamics of face detection in the wild revealed by MVPA. $\mathrm{J}$ Neurosci 34:846-854. CrossRef Medline

Cichy RM, Pantazis D, Oliva A (2014) Resolving human object recognition in space and time. Nat Neurosci 17:455-462. CrossRef Medline

Clarke A, Tyler L (2014) Object-specific semantic coding in human perirhinal cortex. J Neurosci 34:4766-4775. CrossRef Medline

Clarke A, Devereux BJ, Randall B, Tyler LK (2015) Predicting the time course of individual objects with MEG. Cereb Cortex 25:36023612. CrossRef Medline

Coggan DD, Liu W, Baker DH, Andrews TJ (2016) Category-selective patterns of neural response in the ventral visual pathway in the absence of categorical information. Neuroimage 135:107-114. CrossRef Medline

Connolly AC, Guntupalli JS, Gors J, Hanke M, Halchenko YO, Wu Y-C, Abdi H, Haxby JV (2012) The representation of biological classes in the human brain. J Neurosci 32:2608-2618. CrossRef Medline

DiCarlo JJ, Cox DD (2007) Untangling invariant object recognition. Trends Cogn Sci 11:333-341. CrossRef Medline

Downing PE, Jiang Y, Shuman M, Kanwisher N (2001) A cortical area selective for visual processing of the human body. Science 293: 2470-2473. CrossRef Medline

Epstein R, Kanwisher N (1998) A cortical representation of the local visual environment. Nature 392:598-601. CrossRef Medline

Farah MJ (1990) Visual agnosia. Cambridge, MA: MIT.

Freeman J, Simoncelli EP (2011) Metamers of the ventral stream. Nat Neurosci 14:1195-1201. CrossRef Medline
Golomb JD, Kanwisher N (2012) Higher level visual cortex represents retinotopic, not spatiotopic, object location. Cereb Cortex 22: 2794-2810. CrossRef Medline

Haxby JV, Gobbini MI, Furey ML, Ishai A, Schouten JL, Pietrini P (2001) Distributed and overlapping representations of faces and objects in ventral temporal cortex. Science 293:2425-2430. CrossRef Medline

Hochstein S, Ahissar M (2002) View from the top: hierarchies and reverse hierarchies in the visual system. Neuron 36:791-804. Medline

Kanwisher N (2010) Functional specificity in the human brain: a window into the functional architecture of the mind. Proc Natl Acad Sci U S A 107:11163-11170. CrossRef Medline

Kanwisher N, McDermott J, Chun MM (1997) The fusiform face area: a module in human extrastriate cortex specialized for face perception. J Neurosci 17:4302-4311. Medline

Kriegeskorte N, Mur M, Ruff DA, Kiani R, Bodurka J, Esteky H, Tanaka K, Bandettini PA (2008) Matching Categorical Object Representations in Inferior Temporal Cortex of Man and Monkey. Neuron 60:1126-1141. CrossRef Medline

Lamme VA, Roelfsema PR (2000) The distinct modes of vision offered by feedforward and recurrent processing. Trends Neurosci 23:571-579. Medline

Levy I, Hasson U, Avidan G, Hendler T, Malach R (2001) Centerperiphery organization of human object areas. Nat Neurosci 4:533-539. CrossRef Medline

Malach R, Reppas JB, Benson RR, Kwong KK, Jiang $H$, Kennedy WA, Ledden PJ, Brady TJ, Rosen BR, Tootell RBH (1995) Objectrelated activity revealed by functional magnetic resonance imaging in human occipital cortex. Proc Natl Acad Sci U S A 92:8135-8139. CrossRef

Marr D, Hildreth E (1980) Theory of edge detection. Proc R Soc Lond B Biol Sci 207:187-217. Medline

McNeil JE, Warrington EK (1993) Prosopagnosia: a face-specific disorder. Q J Exp Psychol A 46:1-10. Medline

Moscovitch M, Winocur G, Behrmann M (1997) What is special about face recognition? Nineteen experiments on a person with visual object agnosia and dyslexia but normal face recognition. J Cogn Neurosci 9:555-604. CrossRef Medline

Mur M, Kriegeskorte N (2014) What's there, distinctly, when and where? Nat Neurosci 17:332-333. CrossRef Medline

Naselaris T, Prenger RJ, Kay KN, Oliver M, Gallant JL (2009) Bayesian reconstruction of natural images from human brain activity. Neuron 63:902-915. CrossRef Medline

Nili H, Wingfield C, Walther A, Su L, Marslen-Wilson W, Kriegeskorte N (2014) A toolbox for representational similarity analysis. PLoS Comput Biol 10:e1003553 CrossRef

O'Toole AJ, Jiang F, Abdi H, Haxby JV (2005) Partially distributed representations of objects and faces in ventral temporal cortex. J Cogn Neurosci 17:580-590.

Pelli DG (1997) The VideoToolbox software for visual psychophysics: transforming numbers into movies. Spat Vis 10:437-442. Medline

Rice GE, Watson DM, Hartley T, Andrews TJ (2014) Low-level image properties of visual objects predict patterns of neural response across category-selective regions of the ventral visual pathway. $\mathrm{J}$ Neurosci 34:8837-8844. CrossRef Medline

Silson EH, Chan AW-Y, Reynolds RC, Kravitz DJ, Baker Cl (2015) A retinotopic basis for the division of high-level scene processing between lateral and ventral human occipitotemporal cortex. J Neurosci 35:11921-11935. CrossRef

Watson DM, Young AW, Andrews TJ (2016) Spatial properties of objects predict patterns of neural response in the ventral visual pathway. Neuroimage 126:173-183. CrossRef Medline 